\title{
COMPACTNESS IN VECTOR-VALUED BANACH FUNCTION SPACES
}

\author{
JAN VAN NEERVEN
}

\begin{abstract}
We give a new proof of a recent characterization by Diaz and Mayoral of compactness in the Lebesgue-Bochner spaces $L_{X}^{p}$, where $X$ is a Banach space and $1 \leq p<\infty$, and extend the result to vector-valued Banach function spaces $E_{X}$, where $E$ is a Banach function space with order continuous norm.
\end{abstract}

Let $X$ be a Banach space. The problem of describing the compact sets in the Lebesgue-Bochner spaces $L_{X}^{p}, 1 \leq p<\infty$, goes back to the work of Riesz, Fréchet, Vitali in the scalar-valued case, cf. [7, and has been considered by many authors, cf. [2, 4, 5, 11, 12. In a recent paper, Diaz and Mayoral [5] proved that if the underlying measure space is finite, then a subset $K$ of $L_{X}^{p}$ is relatively compact if and only if $K$ is uniformly $p$-integrable, scalarly relatively compact, and either uniformly tight or flatly concentrated. Their proof relies on the Diestel-RuessSchachermayer characterization [6] of weak compactness in $L_{X}^{1}$ and the notion of Bocce oscillation, which was studied recently by Girardi [8] and Balder-GirardiJalby [3] in the context of compactness in $L_{X}^{1}$. The purpose of this note is to present an extension of the Diaz-Mayoral result to vector-valued Banach function spaces $E_{X}$, with a proof based on Prohorov's tightness theorem.

We begin with some preliminaries on Banach lattices and Banach function spaces. Our terminology is standard and follows 9 .

A Banach lattice $E$ is said to have order continuous norm if every net in $E$ which decreases to 0 converges to 0 . Every separable Banach function space $E$ has this property. Indeed, because such spaces are Dedekind complete [9, Lemma 2.6.1] and cannot contain an isomorphic copy of $l^{\infty}$, this follows from [9, Corollary 2.4.3].

A subset $F$ of a Banach lattice $E$ is called almost order bounded if for every $\varepsilon>0$ there exists an element $x_{\varepsilon} \in E_{+}$such that $F \subseteq\left[-x_{\varepsilon}, x_{\varepsilon}\right]+B(\varepsilon)$, where $\left[-x_{\varepsilon}, x_{\varepsilon}\right]:=\left\{y \in E:-x_{\varepsilon} \leq y \leq x_{\varepsilon}\right\}$ and $B(\varepsilon):=\{x \in X:\|x\|<\varepsilon\}$. It follows from [9, Theorem 2.4.2] that every almost order bounded set in a Banach lattice with order continuous norm is relatively weakly compact.

Lemma 1. Let $E$ be a Banach lattice and let $I$ be a dense ideal in $E$. If the set $A \subseteq E^{+}$is almost order bounded, then for every $\varepsilon>0$ there exists an element $x_{\varepsilon} \in I^{+}$such that $A \subseteq\left[0, x_{\varepsilon}\right]+B(\varepsilon)$.

2000 Mathematics Subject Classification. Primary: 46E40, Secondary: 46E30, 46B50, 47D06, 60B05.

Key words and phrases. Compactness, vector-valued Banach function spaces, order continuous norm, almost order boundedness, uniform integrability, tightness.

The author is supported by the 'VIDI subsidie' 639.032.201 in the 'Vernieuwingsimpuls' programme of the Netherlands Organization for Scientific Research (NWO) and by the Research Training Network HPRN-CT-2002-00281. 
Proof. Fix $\varepsilon>0$ and choose $y_{\varepsilon} \in E^{+}$such that $A \subseteq\left[-y_{\varepsilon}, y_{\varepsilon}\right]+B\left(\frac{1}{2} \varepsilon\right)$. Choose $x_{\varepsilon} \in I$ such that $0 \leq x_{\varepsilon} \leq y_{\varepsilon}$ and $\left\|y_{\varepsilon}-x_{\varepsilon}\right\|<\frac{1}{2} \varepsilon$.

Fix $a \in A$, say $a=y+b$ with $y \in\left[-y_{\varepsilon}, y_{\varepsilon}\right]$ and $\|b\|<\frac{1}{2} \varepsilon$. With $z_{\varepsilon}:=y_{\varepsilon}+|b|$ we have $\left\|z_{\varepsilon}-x_{\varepsilon}\right\| \leq\left\|y_{\varepsilon}-x_{\varepsilon}\right\|+\|b\|<\varepsilon$. From $a \leq z_{\varepsilon}$ we infer $\left(a-x_{\varepsilon}\right)^{+} \leq$ $\left(z_{\varepsilon}-x_{\varepsilon}\right)^{+}=z_{\varepsilon}-x_{\varepsilon}$ and hence $\left\|\left(a-x_{\varepsilon}\right)^{+}\right\| \leq\left\|z_{\varepsilon}-x_{\varepsilon}\right\|<\varepsilon$. It follows that $a=a \wedge x_{\varepsilon}+\left(a-x_{\varepsilon}\right)^{+} \in\left[0, x_{\varepsilon}\right]+B(\varepsilon)$.

If $E$ is a Banach function space with order continuous norm, then for all $f \in E$ we have $\lim _{r \rightarrow \infty}\left\|\mathbf{1}_{\{|\phi|>r\}} \phi\right\|_{E}=0$. Motivated by this we shall call a subset $F$ of $E$ uniformly E-integrable if

$$
\lim _{r \rightarrow \infty} \sup _{\phi \in F}\left\|\mathbf{1}_{\{|\phi|>r\}} \phi\right\|_{E}=0 .
$$

For $E=L^{p}$ with $1 \leq p<\infty$, this definition reduces to the classical definition of uniform $p$-integrability.

If $E$ is a Banach function space containing the constant function 1, then every uniformly $E$-integrable subset of $E$ is almost order bounded. From Lemma 1 we deduce the following converse:

Lemma 2. Let $E$ be a Banach function space with order continuous norm over a $\sigma$-finite measure space $(S, \nu)$. If $F \subseteq E^{+}$is almost order bounded, then $F$ is uniformly E-integrable.

Proof. Let $\varepsilon>0$ be fixed. By Lemma 1, applied to $I:=E \cap L^{\infty}(S, \nu)$, we may choose $x_{\varepsilon} \in E^{+}$and real numbers $R_{\varepsilon} \geq 0$ such that $0 \leq x_{\varepsilon} \leq R_{\varepsilon} \nu$-almost everywhere and $F \subseteq\left[0, x_{\varepsilon}\right]+B(\varepsilon)$. Keeping $\phi \in F$ fixed for the moment, we can write $\phi=x+b$ with $x \in\left[0, x_{\varepsilon}\right]$ and $\|b\|_{E}<\varepsilon$. Then, for all $r>0$,

$$
\begin{aligned}
\left\|\mathbf{1}_{\{\phi>r\}} \phi\right\|_{E} & \leq\left\|\mathbf{1}_{\{\phi>r\}} x\right\|_{E}+\left\|\mathbf{1}_{\{\phi>r\}} b\right\|_{E} \\
& \leq\left\|\mathbf{1}_{\left\{x>\frac{1}{2} r\right\}} x\right\|_{E}+\left\|\mathbf{1}_{\left\{|b|>\frac{1}{2} r\right\}} x\right\|_{E}+\|b\|_{E} \\
& \leq\left\|\mathbf{1}_{\left\{x_{\varepsilon}>\frac{1}{2} r\right\}} x_{\varepsilon}\right\|_{E}+\frac{2 R_{\varepsilon}}{r}\|b\|_{E}+\varepsilon,
\end{aligned}
$$

where in the last step we used that $\nu$-almost everywhere we have

$$
0 \leq \frac{1}{2} r \mathbf{1}_{\left\{|b|>\frac{1}{2} r\right\}} x \leq|b| x \leq|b| x_{\varepsilon} \leq R_{\varepsilon}|b| .
$$

The lemma immediately follows from this.

The next lemma gives a sufficient condition for norm convergence in almost order bounded sets. Recall that an element $x^{*} \in E^{*}$ in the dual of a Banach lattice $E$ is called stricly positive if $\left\langle|x|, x^{*}\right\rangle=0$ implies $x=0$.

Lemma 3. Let $E$ be a Banach lattice with order continuous norm and let $F$ be an almost order bounded subset of $E$. If $\left(x_{j}\right)_{j \geq 1}$ is a sequence in $F$ such that $\lim _{j \rightarrow \infty}\left\langle\left|x_{j}\right|, x^{*}\right\rangle=0$ for some strictly positive element $x^{*} \in E^{*}$, then $\lim _{j \rightarrow \infty} x_{j}=$ 0 in $E$.

Proof. Assume the contrary and choose sequences $j_{n} \rightarrow \infty$ and a number $\delta>0$ such that $\left\|x_{j_{n}}\right\|_{E} \geq \delta$ for all $n$. We have

$$
\lim _{m, n \rightarrow \infty}\left\langle\left|x_{j_{m}}-x_{j_{n}}\right|, x^{*}\right\rangle \leq \lim _{m \rightarrow \infty}\left\langle\left|x_{j_{m}}\right|, x^{*}\right\rangle+\lim _{n \rightarrow \infty}\left\langle\left|x_{j_{n}}\right|, x^{*}\right\rangle=0
$$


and therefore, by [10, Lemma 3.8], $\lim _{n \rightarrow \infty} x_{j_{n}}=x$ for some $x \in E$. Then $\|x\| \geq \delta$ and $0=\lim _{n \rightarrow \infty}\left\langle\left|x_{j_{n}}\right|, x^{*}\right\rangle=\left\langle|x|, x^{*}\right\rangle$. This contradicts the fact that $x^{*}$ is strictly positive.

Let $X$ be a Banach space. A set $M$ of Radon probability measures on $X$ is called uniformly tight if for every $\varepsilon>0$ there exists a compact set $K$ in $X$ such that

$$
\mu(K) \geq 1-\varepsilon \quad \forall \mu \in M
$$

By Prohorov's theorem for Radon measures [13, Theorem I.3.6], $M$ is uniformly tight if and only if $M$ relatively weakly compact, i.e., every sequence $\left(\mu_{n}\right)_{n \geq 1}$ has a subsequence $\left(\mu_{n_{k}}\right)_{k \geq 1}$ such that for some Radon probability measure $\mu$ we have

$$
\lim _{k \rightarrow \infty} \int_{X} f d \mu_{n_{k}}=\int_{X} f d \mu \text { for all } f \in C_{b}(X),
$$

where $C_{b}(X)$ is the space of all scalar-valued bounded continuous functions on $X$.

We shall formulate the main result of this paper for Banach function spaces $E$ over a probability space $(\Omega, \mathbb{P})$. This is done merely for convenience; the result extends to arbitrary finite measure spaces by a trivial normalization argument.

The space $E_{X}$ of all strongly $\mathbb{P}$-measurable functions $\phi: \Omega \rightarrow X$ such that $\omega \mapsto\|\phi(\omega)\|$ belongs to $E$ is a Banach space with respect to the norm

$$
\|\phi\|_{E_{X}}:=\|\| \phi\|\|_{E} .
$$

Here, as usual, we identify functions that are equal $\mathbb{P}$-almost everywhere. It follows from [9, Proposition 2.6.3] that $\lim _{n \rightarrow \infty} \phi_{n}=\phi$ in $E_{X}$ implies that for some subsequence we have $\lim _{k \rightarrow \infty} \phi_{n_{k}}(\omega)=\phi(\omega)$ in $X$ for $\mathbb{P}$-almost all $\omega \in \Omega$.

The distribution of a function $\phi \in E_{X}$ is the Radon probability measure $\mu_{\phi}$ on $X$ defined by

$$
\mu_{\phi}(B)=\mathbb{P}\{\phi \in B\} \text { for } B \subseteq X \text { Borel. }
$$

This definition is independent of the representative of $\phi$ used to define $\mu_{\phi}$.

We call a subset $F$ of $E_{X}$ :

- almost order bounded, if $\{\|\phi\|: \phi \in F\}$ is almost order bounded in $E$;

- scalarly relatively compact, if $\left\{\left\langle\phi, x^{*}\right\rangle: \phi \in F\right\}$ is relatively norm compact in $E$ for all $x^{*} \in E^{*}$;

- uniformly tight, if $\left\{\mu_{\phi}: \phi \in F\right\}$ is uniformly tight.

Lemma 4. Let $F$ be a subset of $E_{X}$. If $F$ is almost order bounded, then also $F-F$ is almost order bounded.

Proof. Fix $\varepsilon>0$. Using Lemma 1 we choose $x_{\varepsilon} \in E^{+}$such that $\|\phi\| \in\left[0, x_{\varepsilon}\right]+B\left(\frac{1}{2} \varepsilon\right)$ for all $\phi \in F$.

Step 1 - We claim that each $\phi \in F$ can be written as $\phi=f+g$ with $\|f\| \in\left[0, x_{\varepsilon}\right]$ and $g \in B\left(\frac{1}{2} \varepsilon\right)$. Indeed, we have

$$
\phi=\left(\mathbf{1}_{\left\{\|\phi\| \leq x_{\varepsilon}\right\}} \phi+\mathbf{1}_{\left\{\|\phi\|>x_{\varepsilon}\right\}} \frac{x_{\varepsilon}}{\|\phi\|} \phi\right)+\mathbf{1}_{\left\{\|\phi\|>x_{\varepsilon}\right\}} \frac{\left(\|\phi\|-x_{\varepsilon}\right)}{\|\phi\|} \phi .
$$

For the first term on the right hand side we have

$$
\left\|\mathbf{1}_{\left\{\|\phi\| \leq x_{\varepsilon}\right\}} \phi+\mathbf{1}_{\left\{\|\phi\|>x_{\varepsilon}\right\}} \frac{x_{\varepsilon}}{\|\phi\|} \phi\right\| \in\left[0, x_{\varepsilon}\right] .
$$


Writing $\|\phi\|=a+b$ with $a \in\left[0, x_{\varepsilon}\right]$ and $\|b\|_{E}<\frac{1}{2} \varepsilon$, for the second term we have

$$
\left\|\mathbf{1}_{\left\{\|\phi\|>x_{\varepsilon}\right\}} \frac{\left(\|\phi\|-x_{\varepsilon}\right)}{\|\phi\|} \phi\right\|=\mathbf{1}_{\left\{\|\phi\|>x_{\varepsilon}\right\}}\left(a+b-x_{\varepsilon}\right) \leq \mathbf{1}_{\left\{\|\phi\|>x_{\varepsilon}\right\}} b,
$$

which shows that

$$
\left\|\mathbf{1}_{\left\{\|\phi\|>x_{\varepsilon}\right\}} \frac{\left(\|\phi\|-x_{\varepsilon}\right)}{\|\phi\|} \phi\right\|_{E_{X}} \leq\|b\|_{E}<\frac{1}{2} \varepsilon .
$$

This proves the claim.

Step 2 - Let $\phi_{1}, \phi_{2} \in F$ be given, and write $\phi_{k}=f_{k}+g_{k}$, where $\left\|f_{k}\right\| \in\left[0, x_{\varepsilon}\right]$ and $g_{k} \in B\left(\frac{1}{2} \varepsilon\right)$ for $k=1,2$. Then

$$
\left\|\phi_{1}-\phi_{2}\right\|=\left\|f_{1}-f_{2}\right\|+\left(\left\|\phi_{1}-\phi_{2}\right\|-\left\|f_{1}-f_{2}\right\|\right),
$$

with $\left\|f_{1}-f_{2}\right\| \in\left[0,2 x_{\varepsilon}\right]$ and

$$
\left|\left\|\phi_{1}-\phi_{2}\right\|-\left\|f_{1}-f_{2}\right\|\right| \leq\left\|g_{1}-g_{2}\right\|,
$$

which shows that \|\|$\phi_{1}-\phi_{2}\|-\| f_{1}-f_{2}\|\|_{E}<\varepsilon$.

Theorem 5. Let $E$ be a Banach function space with order continuous norm over a probability space $(\Omega, \mathbb{P})$. Let $X$ a Banach space. For a subset $F$ of $E_{X}$ the following assertions are equivalent:

(1) The set $F$ is relatively compact;

(2) The set $F$ is uniformly tight, almost order bounded, and scalarly relatively compact.

As has been mentioned above, every separable Banach function space has order continuous norm.

Proof. Without loss of generality we may assume that $E$ is saturated, i.e., that $f \equiv 0$ on $A$ for all $f \in E$ implies $\mathbb{P}(A)=0$ [14, Section 67].

(11) $\Rightarrow$ (2): It is clear that the relative compactness of $F$ implies its almost order boundedness and scalar relative compactness.

To prove the uniform tightness of $F$, by Prohorov's theorem it suffices to show that every sequence $\left(\phi_{n}\right)_{n \geq 1}$ in $F$ has a subsequence $\left(\phi_{n_{j}}\right)_{j \geq 1}$ whose distributions converge weakly.

Let us write $\mu_{n}:=\mu_{\phi_{n}}$ for simplicity. Since $F$ is compact we may assume, by passing to a subsequence, that $\left(\phi_{n}\right)_{n \geq 1}$ converges in $E_{X}$ to an element $\phi \in E_{X}$. By passing to a further subsequence we may also assume that the convergence takes place almost surely. Let $\mu:=\mu_{\phi}$ be the distribution of $\phi$. Then for all $f \in C_{b}(X)$ we have, by dominated convergence,

$$
\lim _{n \rightarrow \infty} \int_{X} f d \mu_{n}=\lim _{n \rightarrow \infty} \int_{\Omega} f \circ \phi_{n} d \mathbb{P}=\int_{\Omega} f \circ \phi d \mathbb{P}=\int_{X} f d \mu .
$$

(2) $\Rightarrow$ (11): Let $\left(\phi_{n}\right)_{n \geq 1}$ be a sequence in $F$. We shall prove that some subsequence $\left(\phi_{n_{j}}\right)_{j \geq 1}$ converges in $E_{X}$.

Step 1 - Let $\nu_{n, m}$ denote distribution of the random variable $\phi_{n}-\phi_{m}$. We claim that the family $\left(\nu_{n, m}\right)_{n, m \geq 1}$ is uniformly tight. The proof is standard and runs as follows. Fix some $\varepsilon>0$. Since $\left(\mu_{n}\right)_{n \geq 1}$ is uniformly tight we may choose a compact set $K \subseteq X$ such that $\mu_{n}(K) \geq 1-\varepsilon$ for all $n \geq 1$. The set $L=\{x-y$ : $x, y \in K\}$ is compact as well, being the image of the compact set $K \times K$ under the 
continuous map $(x, y) \mapsto x-y$. Noting that $\phi_{n}(\omega) \in K$ and $\phi_{m}(\omega) \in K$ implies $\phi_{n}(\omega)-\phi_{m}(\omega) \in L$, the claim now follows from

$$
\begin{aligned}
\nu_{n, m}(L) & \geq \mathbb{P}\left\{\phi_{n} \in K, \phi_{m} \in K\right\} \\
& \geq 1-\left(\mathbb{P}\left\{\phi_{n} \in \complement K\right\}+\mathbb{P}\left\{\phi_{m} \in \complement K\right\}\right)=1-\left(\mu_{n}(K)+\mu_{m}(K)\right) \geq 1-2 \varepsilon .
\end{aligned}
$$

Step 2 - Since $F$ is uniformly tight, we may assume $X$ to be separable. Let $\left(x_{m}^{*}\right)_{m>1}$ be a sequence in $X^{*}$ whose intersection with every ball is weak*-dense. As before we let $\mu_{n}$ denote the distribution of $\phi_{n}$. Prohorov's theorem implies the existence of a weakly convergent subsequence $\left(\mu_{n_{j}}\right)_{j \geq 1}$. By passing to a subsequence we may assume that the limit $\psi_{m}:=\lim _{j \rightarrow \infty}\left\langle\phi_{n_{j}}, x_{m}^{*}\right\rangle$ exists in $E$ for all $m$ and that the convergence happens almost surely.

We claim that $\nu_{n_{j}, n_{k}} \rightarrow \delta_{0}$ weakly as $j, k \rightarrow \infty$, where $\delta_{0}$ denotes the Dirac measure concentrated at 0 . Let $j_{l} \rightarrow \infty$ and $k_{l} \rightarrow \infty$. By Step 1 we may pass to a subsequence of the indices $l$ and assume that $\nu_{n_{j_{l}}, n_{k_{l}}} \rightarrow \nu$ for some Radon probability measure $\nu$ on $X$. By taking Fourier transforms, from the almost sure convergence $\lim _{l \rightarrow \infty}\left\langle\phi_{n_{j_{l}}}, x_{m}^{*}\right\rangle=\lim _{l \rightarrow \infty}\left\langle\phi_{n_{k_{l}}}, x_{m}^{*}\right\rangle=\psi_{m}$ we see that for all $m$,

$$
\widehat{\nu}\left(x_{m}^{*}\right)=\lim _{l \rightarrow \infty} \widehat{\nu_{n_{l}, n_{k_{l}}}}\left(x_{m}^{*}\right)=\lim _{l \rightarrow \infty} \int_{\Omega} \exp \left(-i\left\langle\phi_{n_{j_{l}}}-\phi_{n_{k_{l}}}, x_{m}^{*}\right\rangle\right) d \mathbb{P}=1=\widehat{\delta_{0}}\left(x_{m}^{*}\right)
$$

by dominated convergence. Noting that the weak ${ }^{*}$-topology of every ball in $X^{*}$ is metrizable, combined with the fact that the Fourier transforms of Radon probability measures are weak*-sequentially continuous, it follows that $\widehat{\nu}=\widehat{\delta_{0}}$. Therefore $\nu=\delta_{0}$ by the uniqueness of the Fourier transform. Since the sequences $j_{l}$ and $k_{l}$ were arbitrary, this proves the claim.

Step 3 - It remains to show that the sequence $\left(\phi_{n_{j}}\right)_{j \geq 1}$ is Cauchy in $E_{X}$.

For $j, k \geq 1$ define the functions $g_{j k} \in E$ by

$$
g_{j k}:=\left\|\phi_{n_{j}}-\phi_{n_{k}}\right\| \text {. }
$$

For $n \geq 1$ choose $r_{n} \geq 0$ so large that

$$
\left\|\mathbf{1}_{\left\{g_{j k}>r_{n}\right\}} g_{j k}\right\|_{E}<\frac{1}{n} \text { for all } j, k \geq 1 .
$$

This is possible since $F-F$ is almost order bounded by Lemma 4, By Lemma 2, $\|F-F\|$ is uniformly $E$-integrable.

Let $f \in C_{b}(\mathbb{R})$ be arbitrary. By Step 2 and Prohorov's theorem,

$$
\lim _{j, k \rightarrow \infty} \int_{\Omega} f \circ g_{j k} d \mathbb{P}=f(0)
$$

Keeping $n \geq 1$ fixed for the moment and taking $f(t)=|t| \wedge r_{n}$, it follows that there exists an index $N_{n} \geq 1$ such that

$$
\int_{\Omega} g_{j k} \wedge r_{n} d \mathbb{P}<\frac{1}{n} \text { for all } j, k \geq N_{n} .
$$

Let $0 \leq \psi_{0} \leq \mathbf{1}$ be a $\mathbb{P}$-almost everywhere strictly positive function belonging to the associate space $E^{\prime}$, which is defined as the space of all $\nu$-measurable functions $\psi$ on $S$ such that

$$
\|\psi\|_{E^{\prime}}:=\sup _{\|\phi\|_{E} \leq 1} \int_{\Omega}|\phi \psi| d \mathbb{P}<\infty .
$$

Such a function exists since $E$ is assumed to be saturated. Note that $\psi_{0}$ is strictly positive as element of $E^{*}$. For $j, k \geq N_{n}$,

$$
0 \leq\left\langle g_{j k}, \psi_{0}\right\rangle \leq\left\langle g_{j k} \wedge r_{n}, \psi_{0}\right\rangle+\left\langle\mathbf{1}_{\left\{g_{j k}>r_{n}\right\}} g_{j k}, \psi_{0}\right\rangle<\frac{1}{n}\left(1+\left\|\psi_{0}\right\|_{E^{\prime}}\right) .
$$


It follows that $\lim _{j, k \rightarrow \infty}\left\langle g_{j k}, \psi_{0}\right\rangle=0$. Now Lemma 3 shows that $\lim _{j, k \rightarrow \infty} g_{j k}=0$ in $E$.

As in [5, the uniform tightness assumption in assertion (2) may be replaced by flat concentration. This follows from Prohorov's theorem in combination with the well known result of de Acosta [1, see also [13, Theorem I.3.7], that a family $M$ of Radon probability measures on $X$ is uniformly tight if and only if $M$ is flatly concentrated and for all $x^{*} \in E^{*}$ the set of image measures $\left\langle M, x^{*}\right\rangle=\left\{\left\langle\mu, x^{*}\right\rangle\right.$ : $\left.x^{*} \in E^{*}\right\}$ is uniformly tight.

\section{REFERENCES}

[1] A. DE Acosta, Existence and convergence of probability measures in Banach spaces, Trans. Am. Math. Soc. 152 (1970), 273-298.

[2] J.-P. Aubin, Un théorème de compacité, C. R. Acad. Sci. Paris 256 (1963), 5042-5044.

[3] E.J. Balder, M. Girardi, And V. Jalby, From weak to strong types of $L_{E}^{1}$-convergence by the Bocce criterion, Studia Math. 111 (1994), 241-262.

[4] J.K. Brooks and N. Dinculeanu, Conditional expectations and weak and strong compactness in spaces of Bochner integrable functions, J. Multivariate Anal. 9 (1979), 420-427.

[5] S. Diaz and F. Mayoral, On compactness in spaces of Bochner integrable functions, Acta Mat. Hungar. 83 (1999), 231-239.

[6] J. Diestel, W.M. Ruess, W. Schachermayer, On weak compactness in $L^{1}(\mu, X)$, Proc. Amer. Math. Soc. 118 (1993), 447-453.

[7] N. Dunford and J.T. Schwartz, "Linear Operators, Part I", Pure and Applied Mathematics, Vol. 7, Interscience Publishers, Inc., New York, 1958.

[8] M. Girard, Weak versus norm compactness in $L_{1}$ : the Bocce criterion, Studia Math. 98 (1991), 95-97.

[9] P. Meyer-Nieberg, "Banach Lattices", Universitext, Springer-Verlag, 1991.

[10] F. RÄBiger, Stability and ergodicity of dominated semigroups. II. The strong case, Math. Ann. 297 (1993), 103-116.

[11] R. Rossi and G. Savaré, Tightness, integral equicontinuity and compactness for evolution problems in Banach spaces, Ann. Sc. Norm. Super. Pisa Cl. Sci. 2 (2003), 395-431.

[12] J. Simon, Compact sets in the space $L^{p}(0, T ; B)$, Ann. Mat. Pura Appl. 146 (1987), 65-96.

[13] N.N. Vakhania, V.I. Tarieladze and S.A. Chobanyan, "Probability Distributions on Banach Spaces", D. Reidel Publishing Company, Dordrecht-Boston-Lancaster-Tokyo, 1987.

[14] A.C. ZaAnen, "Integration", North Holland, Amsterdam, 1967.

Delft Institute of Applied Mathematics, Technical University of Delft, P.O. Box 5031, 2600 GA Delft, The Netherlands

E-mail address: J.vanNeerven@math.tudelft.nl 